\title{
Adenocarcinoma in Orthotopic Neobladder 19 Years After Radical Cystoprostatectomy
}

\author{
Orkunt Ozkaptan ${ }^{1}$, Alkan Cubuk², Erdinc Dincer ${ }^{1}$ and Osman Murat Ipek ${ }^{1}$ \\ ${ }^{1}$ Department of Urology, Kartal Dr. Lutfi Kirdar Traning and Research Hospital, Istanbul, Turkey \\ ${ }^{2}$ Department of Urology, Kırklareli University School of Medicine, Kırklareli, Turkey
}

\begin{abstract}
Radical cystectomy and orthotopic neobladder are the most preferred surgical approaches for the treatment of invasive bladder cancer. Secondary tumour of ileum on neobladder is very rare. We report a 67-year man complaining of hematuria with the history of ileal neobladder for 19 years. An ileal adenocarcinoma of neobladder was detected with transurethral resection. He also had a non-functioning right kidney. A neobladderectomy. with right nephroureterectomy and ureterocutaneostomy was performed. An adenocarcinoma invading subserosal layer was reported. At six months postoperatively, patient was free of recurrence and progression. Seconder tumour of neobladder is a very rare condition. Early diagnosis and excision of neobladder may provide cure.
\end{abstract}

Key Words: Bladder cancer, Urinary diversion, Cystectomy, lleum, Adenocarcinoma.

How to cite this article: Ozkaptan O, Cubuk A, Dincer E, Ipek OM. Adenocarcinoma in Orthotopic Neobladder 19 Years After Radical Cystoprostatectomy. J Coll Physicians Surg Pak 2021; 31(05):588-590.

\section{INTRODUCTION}

Radical cystectomy is the mainstay of treatment for muscle-invasive and high-grade non-invasive bladder cancer. After radical cystectomy, some form of urinary diversion is required. Currently, using the ileum for urinary diversion is the most common method. Nowadays, orthotopic neobladder is the preferred type of diversion, especially in young patients without comorbidities. ${ }^{1}$ The ileal neobladder, which provides good continence and voiding habits with relatively low complications, is one of the most commonly performed procedures among different types of orthotopic neobladder substitutions. ${ }^{2}$ This report presents a man who developed adenocarcinoma in the ileal neobladder after radical cystoprostatectomy with neobladder reconstruction.

\section{CASE REPORT}

A 67-year man, who had undergone a radical cystoprostatectomy with a Studer ileal neobladder for stage pT2NOMO transitional cell carcinoma of the bladder 19 years ago, was referred to our clinic for intermittent macroscopic hematuria. The patient was under followup until seven years after surgery and interrupted routine visits thereafter. On anamnesis, the patient reported having intermittent haematuria starting six months ago.

Correspondence to: Dr. Alkan Cubuk, Department of Urology, Kartal Dr. Lutfi Kirdar Traning and Research Hospital, Istanbul, Turkey

E-mail: alkancubuk@hotmail.com

Received: August 31, 2019; Revised: November 18, 2020; Accepted: December 10, 2020

DOI: https://doi.org/10.29271/jcpsp.2021.05.588
Laboratory examinations, including routine blood count, renal function tests, and urine tests revealed anaemia (haemoglobin $(\mathrm{Hb}): 10.1 \mathrm{~g} / \mathrm{dl}$ ), a slightly increased creatinine level ( $1.4 \mathrm{mg} / \mathrm{dl})$, and blood in the urine. A magnetic resonance urography showed a $48 \mathrm{~mm}$ mass on the left posterior wall of the neobladder (Figure 1). There were no signs of a tumour in the upper urinary tract; further, no evidence for lymph node metastasis and solid organ metastasis was seen on radiological evaluation. Additionally, a non-functioning kidney was detected with Tc-99m dimercaptosuccinic acid (DMSA) on the right side 10 years ago; and due to high risk of peroperative morbidity, surgery was not performed.

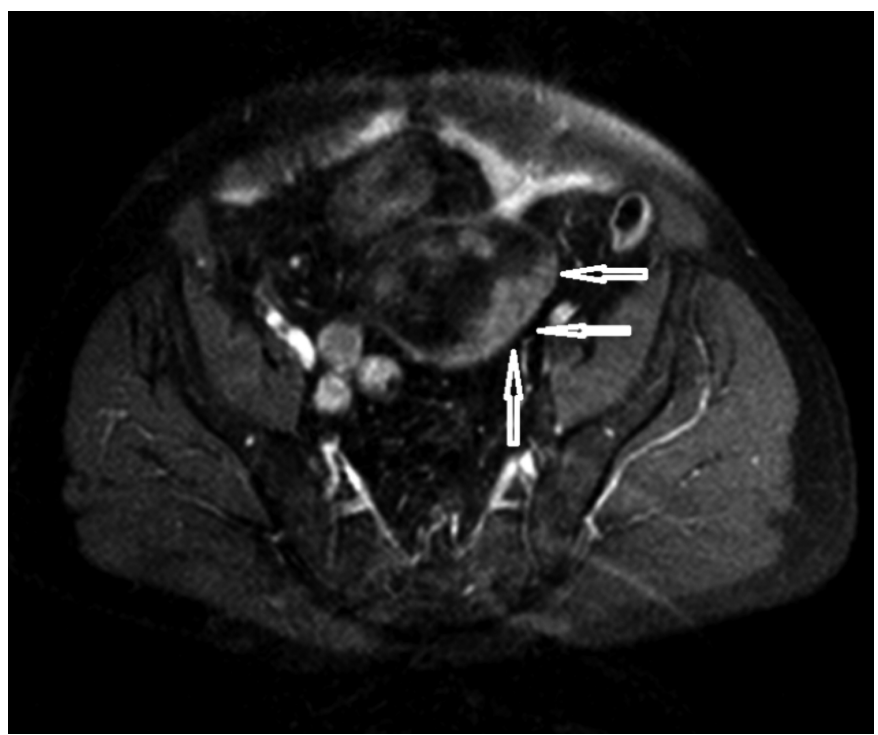

Figure 1: Pelvic MRI axial view demonstrates mass on the left posterior wall of neobladder (arrow). 
Endoscopic evaluation under general anaesthesia was planned for the patient. The urethra was totally normal. An approximately $5 \mathrm{~cm}$ large solid mass on the posterior wall of the neobladder was observed and removed by transurethral resection in the same session. Histopathological examination revealed an adenocarcinoma of the ileum. An open surgical treatment including excision of the ileal neobladder, right nephroureterectomy and left ureterocutaneostomy was performed (Figure 2). The postoperative course of the patient was uneventful. Pathological examination of the specimen confirmed the diagnosis of adenocarcinoma of the ileum invading the subserosal layer (extracellular mucin + , Figure 3). The nephroureterectomy specimen was tumour-free. The patient was referred to oncologists for planning further treatment modalities. At sixth month of follow-up, no evidence of recurrence or metastasis was found.

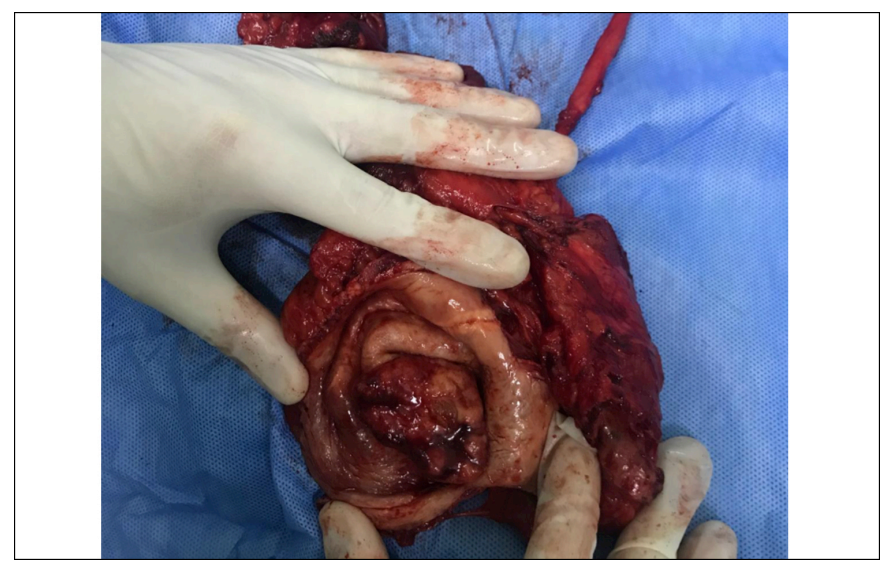

Figure 2: Neobladderectomy + nephroureterectomy specimen. Arrow shows tumouron neobladderwall.

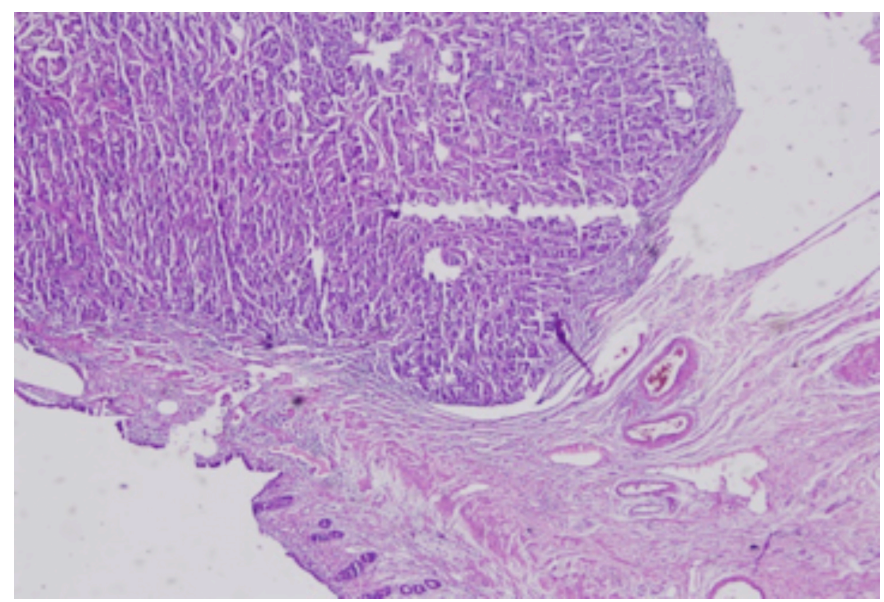

Figure 3. Microscopic view of neobladder showing adenocarcinoma (hematoxylin and eosin, $\times 40$ ).

\section{DISCUSSION}

The trueincidence ofintestinaladenocarcinomain urinary diversions after radical cystectomy is not well known. The incidence of secondary tumours in the ileal neobladder and ileal conduit was reported as $0.05 \%$ and $0.02 \%$, respectively by Kälble et al. ${ }^{3}$ A small number of case reports is available in the literature. The present case report is the first neobladder adenocarcinoma case of our series up to now.
Pathogenesis of neobladder carcinogenesis is still incompletely understood. Moyer et al. suggested tobacco-derived urinary carcinogens bathing the epithelium as the main pathway since most patients continue to smoke after cystectomy. ${ }^{4}$ Austen et al. speculated that factors in urine that promote native bladder cancer also promote secondary tumours after urinary diversion. ${ }^{5}$ However, Qubaiah et al. reported that it is unknown whether tobacco-derived carcinogens are responsible for carcinoma of the small intestine. ${ }^{6}$ In our case, the patient had no family history of small intestinal cancer; however, he was a tobacco smoker.

Ali-el-Dein et al. reported the incidence of secondary tumours in ileocystoplasties to be 5.5\%; whereas, the incidence for ileal conduit was found as $0.3 \%{ }^{7}$ The risk of secondary tumours in ileal conduit and ileal neobladder was similar according to Austen etal. ${ }^{5}$

The mean survival duration for patients who have received curative-intent treatment for bladder cancer is increasing. As a result, the risk of developing cancer in a urinary diversion is also increasing. The latency periods of secondary tumours in orthotopic neobladder vary up to 20 years. ${ }^{8}$ However, it is not possible to define an exact latency period for the occurrence of these tumours.

Ali-el-Dein et al. reported that secondary malignancies in ileal conduits develop in ileoureteral anastomosis while tumours in colonic neobladders occur distant from ureterointestinal anastomosis. ${ }^{7}$ Tumour localisation of our case was near the right ureteric orifice, and that was comparable with the literature.

Commonsigns formalignancyare haematuria, ureteralobstruction, dysuria and pyuria. The patient of the current case report was referred with haematuria and dysuria. Non-invasive diagnostic tools are insufficient for differential diagnosis. In patients with haematuria and recurrent infection, endoscopic evaluation of the neobladder should be used. Pickard et al. advised routine urinary cytological examination for all patients for screening at least yearly, starting four years after surgery. ${ }^{9}$

In conclusion, tumour development in ileal neobladder should be kept in mind for patients suffering from haematuria. Endoscopic evaluation of neobladder is essential. Total removal of neobladder is the curative treatment option.

\section{AKNOWLEDGEMENT:}

We would like to thank Dr. Oktay Akca for his contributions during the operation of this case.

\section{CONFLICT OF INTEREST:}

Authors declared no conflict of interest

\section{PATIENT'S CONSENT:}

The consent of the patient was taken prior to the writing of the manuscript.

\section{AUTHORS' CONTRIBUTION:}

O०: Concept, design, supervision, writer.

AC: Concept, supervision, writer, literature review, critical review. 
ED: Data collection, material.

OMI: Data collection, material, analysis and interpretation.

\section{REFERENCES}

1. Fisch $M$, Thüroff JW. Continent cutaneous diversion. BJU Int 2008; 102(9b):1314-9. doi: 10.1111/j.1464-410X. 2008.07976.x.

2. Nazim SM, Ather MH, Abbas F. Functional and clinical outcome of ileal (Studer) neo-bladder reconstruction: Single centre experience from Pakistan. J Coll Physicians Surg Pak 2014; 24(8):586-90.

3. Kälble T, Hofmann I, Riedmiller H, Vergho D. Tumour growth in urinary diversion: A multicenter analysis. Eur Urol 2011; 60(5):1081-6. doi: 10.1016/j.eururo.2011. 07.006 .

4. Moyer GC, Grubb RL, Johnson FE. Intestinal adenocarcinoma arising in urinary conduits. Oncol Rep 2012; 27(2):371-5.

5. Austen M, Kalble T. Secondary malignancies in different forms of urinary diversion using isolated gut. J Urol 2004; 172(3):831-8. doi: 10.1097/01.ju.0000134890.07434. 8 e.

6. Qubaiah O, Devesa SS, Platz CE, Huycke MM, Dores GM: Small intestinal cancer: A population-based study of incidence and survival patterns in the United States, 1992 to 2006. Cancer Epidemiol Biomarkers Prev 2010; 19(8):1908-18. doi: 10.1158/1055-9965.EPI-10-0328.

7. Ali-El-Dein B, El-Tabey N, Abdel-Latif M, Abdel-Rahim M, El-Bahnasawy MS. Late uro-ileal cancer after incorporation of ileum into the urinary tract. J Urol 2002; 167(1):84-8.

8. Berberian JP, Goeman L, Allory Y, Abbou CC, Salomon L. Adenocarcinoma of ileal neobladder 20 years after cystectomy. Urology 2006; 68(6): 1343.e9-10. doi: 10.1016/j.urology.2006.09.014

9. Pickard R. Tumour formation within intestinal segments transposed to the urinary tract. World J Urol 2004; 22(3):227-34. doi: 10.1007/s00345-004-0438-4. 